\title{
On the shape of bistable creased strips
}

\author{
Martin G. Walker ${ }^{1, *}$, Keith A. Seffen ${ }^{1}$ \\ Cambridge University Engineering Department \\ Trumpington St, Cambridge, United Kingdom, CB2 1 PZ
}

\begin{abstract}
We investigate the bistable behaviour of folded thin strips bent along their central crease. Making use of a simple Gauss mapping, we describe the kinematics of a hinge and facet model, which forms a discrete version of the bistable creased strip. The Gauss mapping technique is then generalised for an arbitrary number of hinge lines, which become the generators of a developable surface as the number becomes large. Predictions made for both the discrete model and the creased strip match experimental results well. This study will contribute to the understanding of shell damage mechanisms; bistable creased strips may also be used in novel multistable systems.
\end{abstract}

Keywords: creasing, folding, snap-through, bistable, localisation, origami

\section{Introduction}

Studies on the deformation of strips with a curved [1] or folded [2] crosssection have only captured smooth deformation of the strip axis. When the cross-section of the strip consists of a sharp crease or fold, new singular bistable behaviour occurs, which is not revealed by previous models. We address this need here.

In their study of bistable creased disks, Lechenault and Adda-Bedia [3] fold a thin disk about equi-spaced radii, in order to create rotationally symmetrical

\footnotetext{
* Corresponding author

Email addresses: mgw39@cam.ac.uk (Martin G. Walker), kas14@cam.ac.uk (Keith A. Seffen)
}

Preprint submitted to Elsevier

November 18, 2017 
creases. For two or more creases, a central vertex is formed initially, which can be inverted by turning the disk inside out. This configuration is usually bistable, with gently curved material in between creases that have not opened or closed any further. Assuming inextensible i.e. developable deformation, and rigidly-fixed crease angles, they calculate the shapes of the initial and inverted states. Their simplest case is a singly creased disk or strip, which can be readily made using, say, paper card of reasonable stiffness, such as a beer mat: after flexing a few times to establish a crease, it can be "pushed through" to form a vertex, where it maintains inversion. We can do the same in Fig. 1 using a plastically folded metal strip. The observed deformation always has two planes of symmetry centred around the central vertex. It is clear the deformed shape shares features with familiar, if unwelcome, indentations straddling fold lines in car body panels, see Fig. 2. This study therefore applies more broadly to folded or pressed thin-walled structures, typically made of metal and used as skins in vehicles, building cladding, etc.

Because of the developable assumption, infinite material strains and, hence, stresses and strain energy density occur at the vertex in theory. In practice, these are limited by localised yielding and stretching close to the vertex but not extensively if the disk is relatively thin. This begs an obvious question about how bistability is predicated upon formation of the vertex. As a simple counterexample, consider when a small perforation or hole is made where the vertex would appear, see Fig. 1. The effort needed to push through and invert the strip is now less compared to one of similar size without a hole, and bistability remains, as later experiments attest. There are also no discernible differences in shape, which could be scrutinised, for example, by modifying the analysis in [3] to include a central hole.

We choose instead a discrete kinematical formulation in which the deformed shape is approximated by rigid facets folding about hinge lines in the original strip; the central crease is also a hinge line but of fixed rotation. In the limit of a large number of hinges we approach the continuum framework but this is not essential nor analytically efficient. As shown originally in [4] for the familiar "d- 
Cone" and then in [5] for general conical defects, the set of compatible rotations for the least number of viable hinges have a unique geometrical solution when the vertex is assumed to be developable; the resulting folded shape has remarkably similar overall properties compared to the continuous example it represents. When the apex of the vertex is removed by making a hole, the formulation also applies if we assume that hinge lines intersect at a "virtual" vertex at its centre.

The system is kinematically indeterminate for more hinge lines. If we assume that some form of elastic bending is represented by folding, we may extract, hopefully, a single set of unique rotations that also satisfy equilibrium. We therefore construct the "equivalent" strain energy of bending stored by discrete hinge lines before minimising under the developable vertex constraint. When the vertex hole is reduced in size to zero, the level of strain energy approaches infinity, as noted, just as infinite vertex stresses emerge from the continuum analysis in [3], which seem unsatisfactory. However our equilibrium analysis leads to coupled differential equations in the rotations that behave in a bounded manner irrespective of the size of the hole, even if the hole is reduced to zero. In other words, infinite strain energy (and stresses) are largely irrelevant and geometry dominates the nature of solution. This emergent property of the formulation with a hole, we believe, makes an alternative contribution to how such problems are tackled.

The layout is as follows. We first describe the simplest rigid-facet model of a singly-creased strip, where we introduce the Gauss mapping technique for a developable vertex behaviour. We then extend our approach to an arbitrary number of hinge lines, and furnish a worked example to demonstrate a vanishing hole size. Three sets of experimental data are presented and compared to theoretical predictions of the overall shape. When a vertex hole is present, a twofold paradox emerges: the shape of the strip is largely unaffected by the size of the hole even though theory would suggest some differences, and that all specimens appear to conform to the prediction for no hole. We offer a possible explanation but we not do not formally resolve matters before concluding our study. Note that by the nature of our analysis we cannot predict whether or 


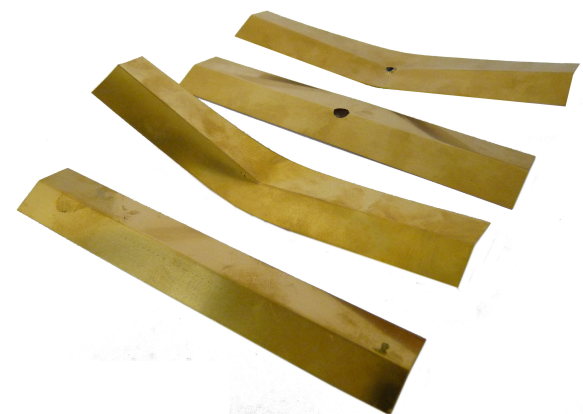

Figure 1: Creased strips with and without a hole in their initial and inverted states. They are made from copper beryllium of thickness $0.1 \mathrm{~mm}$, are $50 \mathrm{~mm}$ wide and $200 \mathrm{~mm}$ long.

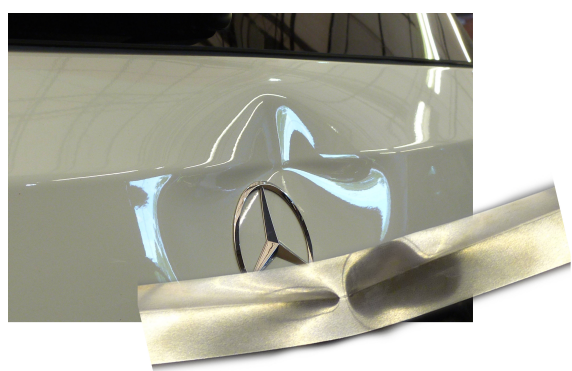

Figure 2: An inverted strip compared to a "dent" in a vehicle body. Note the similarity of vertex shapes lying on the original crease lines.

not the inverted configurations are robustly stable but we do give general limits informed by experiments.

\section{Kinematic Analysis}

\subsection{Gauss Mapping}

We construct two phenomenological models of rigid facets connected by straight hinge lines. The deformation is assumed to be doubly symmetric with respect to the crease axis and the transverse centre line of the strip. Nonsymmetric deformations may be possible depending on the hinge line geometry but the bistable behaviour we see always forms a doubly symmetric shape. Consider first a creased strip with a pair of orthogonal hinge lines as shown in Fig. 3a.

When the strip is bent along the crease axis, the crease must flatten completely before rotations about the hinge lines can occur. The second model shown in Fig. 3b has four hinge lines and six facets. It has a second compatible state which is clearly a simplified version of the inverted shape shown in Fig. 1: there is well defined central vertex under a fixed crease angle.

All possible shapes must satisfy rigid folding compatibility of the hinge lines, which can be enforced through a simple Gauss mapping technique [6]. We map the unit normal vector of each facet to the centre of a unit sphere. As the facets 


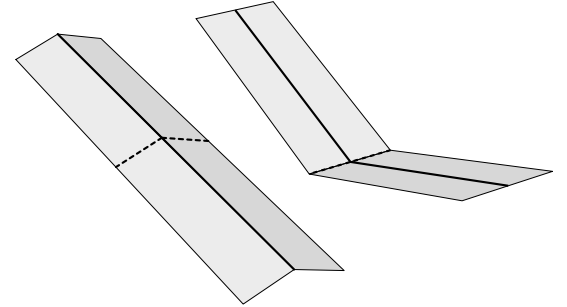

(a)

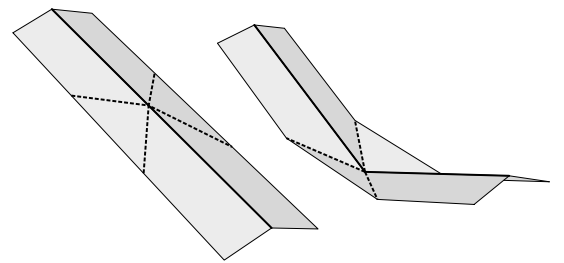

(b)

Figure 3: Phenomenological hinge models with (a) two and (b) four hinge lines (dashed) in their initial and deformed shapes; the central crease line is shown solid. (a) The crease flattens completely and a single central fold forms. (b) A central vertex forms under a fixed crease angle.

rotate, their normal vectors trace out arcs of great circles on the surface of the sphere, with lengths equal to the relative rotation angles between the facets across hinge lines [7]. The signed area enclosed by these arcs is equal to the angular defect at the vertex, which measures the solid angle and, hence, the Gaussian curvature of the vertex [8]. For rigid facets that fold without tearing or crumpling, there can be no defect and thus, the enclosed area must equal zero.

To illustrate how this method works consider the simple two-hinged model shown in Fig. 3a. The facet labelling is shown in Fig. 4a. The rigid facets (A,B,C,D) are labelled according to Bow's notation [9], while the hinge rotation directions satisfy the right-hand rule. The general Gauss mapping is shown in Fig. 4b, which does not correspond to any developable state shown in Fig. 3a. To satisfy the requirement for zero enclosed area, there are two possible cases: $a b=d c=0$, which is the initial state, or $\beta=0$, a flattened crease. Since the possible shapes for this configuration of hinges do not correspond to the observed bistable behaviour, more hinges are needed to capture the observed behaviour.

The hinge line layout of Fig. 3b is formalised in Fig. 5. Each of the four hinge lines are symmetrically separated from the crease line by the same angle $\alpha$, which affords equal rotations, $\theta$, in the deformed case. The six facets (A,B,C,D,E,F) yield a mapping with two crossover points and three enclosed areas, $S_{1}, S_{2}, S_{3}$, 


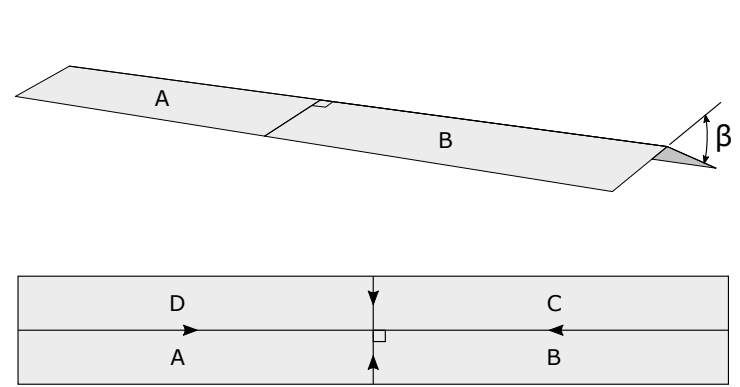

(a) Hinge model

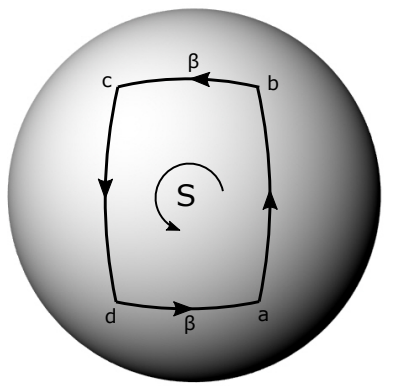

(b) Gauss mapping

Figure 4: (a) Two hinge line model; (b) its Gauss mapping onto the unit sphere of enclosed area $S$.

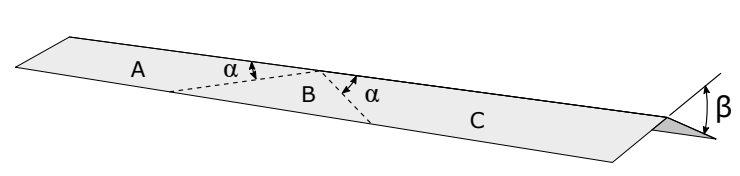

\begin{tabular}{|c|c|c|}
\hline $\mathrm{F}$ & $\alpha \uparrow, \mathrm{E}, \nabla \alpha$ & $\mathrm{D}$ \\
\hline$A$ & 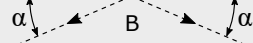 & C \\
\hline
\end{tabular}

(a) Hinge model

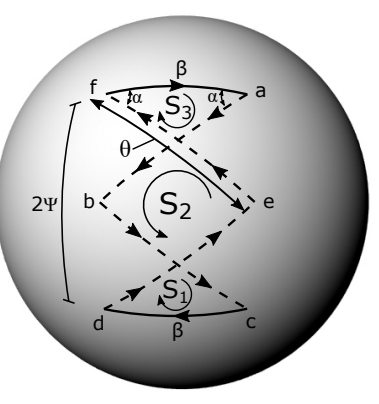

(b) Gauss mapping

Figure 5: (a) Four hinge line model. (b) Its Gauss mapping where rotations $a b, b c$, de, and ef all equal $\theta$; the crease angle, $\beta$, is fixed. The enclosed areas are $S_{1}, S_{2}$ and $S_{3}$. 
which sum to zero for a developable folded shape. For each area, a right-handed orientation in the sense of following the vectors is declared positive, and vice versa. Obviously $a b=b c=d e=e f=0$ gives zero area for $a f=c d=\beta$, which is simply the initial strip layout. The second and only non-trivial solution has $a b=b c=d e=e f=\theta$ and obviates the following exact relationship between $\theta, \alpha$ and $\beta$, which is found by calculating the areas using spherical geometry (Appendix A):

$$
\tan \frac{\theta}{2}=\frac{\tan (\beta / 2)}{\cos \alpha}
$$

We are interested, in particular, in the strip end rotation, which is defined as $\Psi$ in Fig. 6 and provides a means of comparison to [3] and experiments. The distance between lines af and $c d$ in the Gauss mapping, which correspond to the crease line segments between facets $\mathrm{AF}$ and $\mathrm{CD}$, respectively, is the relative rotation of the crease line segments, or $2 \Psi$. Therefore, $\Psi$ depends on the facet rotation, $\theta$, but we can eliminate this using Eqn 1 (see Appendix A) in order to return an expression written purely in terms of the fixed parameters, $\beta$ and $\alpha$ :

$$
\sin \frac{\Psi}{2}=\frac{\sin \alpha \tan (\beta / 2)}{\sqrt{\cos ^{2} \alpha+\tan ^{2}(\beta / 2)}}
$$

There are no other developable states since other rotations yield a Gauss mapping with net area, which implies stretching of the facets at the vertex as the strip is deformed beyond its initial configuration. As the strip approaches its final developable state and the vertex forms, stretching cannot accumulate and must be relieved. Whilst this leads to possible damage in practice if peak in-plane stresses are high enough, their mitigation by non-linear changes in geometry is a familiar pre-requisite for bistable behaviour e.g. the snap-through of shallow arch beams [9]. We cannot however prove as such since our model does not capture the intervening deformation; we can only assess the accuracy of Eqn 2 by comparing it to inverted shapes in practice, which we do in Section 3.

\subsection{Approaching the continuous case}

The Gauss mapping gives a unique solution for the least number of hinge lines. This may be sufficient to capture the gross shape fairly well but more 


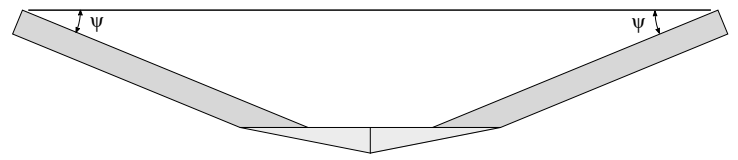

Figure 6: Definition of end rotation angle, $\Psi$, of a deformed strip measured relative to its initial crease line; this also applies to experimental specimens.

lines offer more accuracy and a better description of the shape around the vertex. Since the mapping is a statement of geometrical compatibility for a single developable vertex, it returns only one equation in all of the hinge rotations. Extra solution information is now garnered from an equilibrium scheme.

A strip with width $2 w$ and length $2 l$ is assumed. There are $4 n$ hinge lines in total, with $n$ hinge lines confined to each symmetrical quadrant and separated by equal subtended angles, $\alpha=\pi /(2 n+1)$, as shown in Fig. 7 . Within each quadrant, the hinge line rotations are different and equal to $\theta_{i}$, and repeated fourfold for all quadrants. All hinge lines radiate outwards from a virtual vertex at the centre of a hole of small, but finite, radius $R$. A Gauss mapping assuming a single virtual vertex is applicable despite the hole since $R$ is small and is ultimately reduced to zero for a real vertex.

The adjunct equilibrium behaviour is derived from equivalent strain energy considerations when the hinge lines perform as elastic elements. Rather than endow the hinge line as a conventional torsional spring [10], we spread this rotation over a sector angle $\alpha$, shown shaded in Fig. 7, in the form of a conical section. When the hinge lines are each treated in this manner and assembled together they form a continuous, developable, surface. The corresponding transverse curvature, $c$, is approximately the hinge-line rotation divided by the local width of strip and, if we assume simple linear elastic behaviour, the strain energy density per unit area is $D c^{2} / 2$ [7], where $D$ is the flexural rigidity. First, we isolate the hole from within the strip by enclosing it within a disk of radius $w$, shown dotted in Fig. 7.

Inside the disk, the hinge line rotation per sector admits an equivalent conical curvature at radial distance, $r$, equal to $\theta_{i} / r \alpha$. The strain energy density follows 


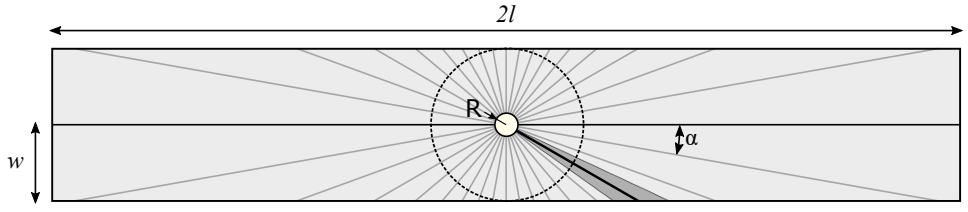

Figure 7: Generalised hinge line model with a central hole of radius $R$ and strip length $2 l$. The disk region (dashed) has radius $w$, and the generator lines subtend equal angles of $\alpha$. A sector relating to a single hinge line is highlighted.

easily, which is then integrated over the area of each sector before summing over $4 n$ sectors to give the equivalent total strain energy of conical bending, $U_{1}$, as:

$$
U_{1}=4 \int_{R}^{\mathrm{w}} \sum_{i=1}^{n} \frac{D}{2}\left(\frac{\theta_{i}}{r \alpha}\right)^{2} r \alpha \mathrm{d} r=\frac{2 D}{\alpha} \ln \left(\frac{\mathrm{w}}{R}\right) \sum_{i=1}^{n} \theta_{i}^{2}
$$

Beyond the disk, the energy is similarly calculated but where the upper integration limit, $L_{i}$, is now the total radial length of hinge $i$ :

$$
U_{2}=4 \sum_{i=1}^{n} \int_{\mathrm{w}}^{L} \frac{D}{2}\left(\frac{\theta_{i}}{r \alpha}\right)^{2} r \alpha \mathrm{d} r=\frac{2 D}{\alpha} \sum_{i=1}^{n} \ln \left(\frac{L_{i}}{w}\right) \theta_{i}^{2}
$$

such that

$$
L_{i}=\min \left(\frac{\mathrm{w}}{\sin i \alpha}, \frac{l}{\cos i \alpha}\right)
$$

depending on where the hinge line terminates. The total strain energy, $U=$ $U_{1}+U_{2}$, can now be minimised for equilibrium configurations of $\theta_{i}$ subject to zero net area of the Gauss mapping. One approach involves first solving for $\theta_{1}$ in terms of the other rotations from the Gauss mapping, substituting into $U$ and then differentiating with respect to each of the remaining $\theta_{i}$. This gives $n-1$ equations of the form:

$$
\frac{\partial U}{\partial \theta_{j}}=\sum_{i=1}^{n} \ln \left(\frac{L_{i}}{R}\right) \theta_{i} \frac{\partial \theta_{i}}{\partial \theta_{j}}=0 \quad \text { for } j \in \mathbb{Z}: 2 \leq j \leq n
$$

Noting that $\partial \theta_{i} / \partial \theta_{j}=0$ for $i \neq j$, and that $\theta_{1}$ is a function of the remaining $\theta_{i}$ due to the zero net area constraint, Eqns 6 become:

$$
\frac{\ln L_{1}-\ln R}{\ln L_{j}-\ln R} \theta_{1} \frac{\partial \theta_{1}}{\partial \theta_{j}}+\theta_{j}=0 \quad \text { for } j \in \mathbb{Z}: 2 \leq j \leq n
$$

Alternatively, the zero net area constraint could be enforced using a Lagrange multiplier which yields an equivalent result. In the limit of $R \rightarrow 0$ for no hole, 

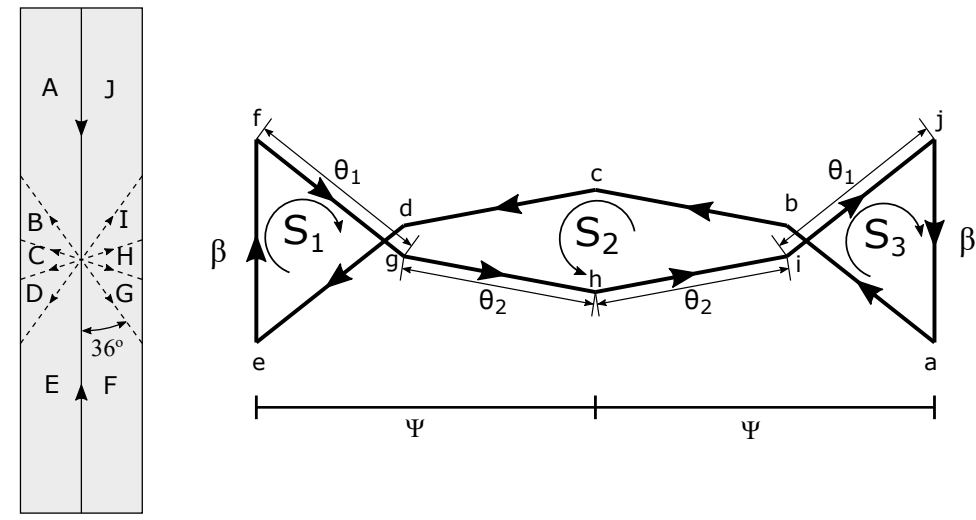

Figure 8: Eight hinge line model and its planar Gauss mapping.

the coefficient of the first term in Eqn 7 becomes unity for all $j$ :

$$
\theta_{1} \frac{\partial \theta_{1}}{\partial \theta_{j}}+\theta_{j}=0 \quad \text { for } j \in \mathbb{Z}: 2 \leq j \leq n
$$

and the strip width, $w$, and length, $l$, do not feature i.e. the inverted shape is independent of planform geometry. The reason is that as $R$ tends to zero, $U_{1}$ becomes much larger than $U_{2}$ in $U$, and the equilibrium solution is dominated by the performance of the inscribed disk region: the deformed shape should therefore approach the continuum solution in [3] for large $n$. Conversely, as $R$ increases, the coefficient of the first term in Eqn 7 is larger than unity and different for each value of $j$, which promotes $U_{2}$ over $U_{1}$. Note also that $D$ is absent, signifying independence from material properties.

As a worked example, consider the creased strip with eight hinges $\left(\alpha=36^{\circ}\right)$ in Fig. 8. From symmetry of the Gauss mapping, the hinge line rotations $a b$, $d e, f g$, and $i j$ are equal to $\theta_{1}$, and $b c, c d, g h$ equal to $\theta_{2}$. For this example we assume that $\beta$ and the hinge rotations are small enough to render the Gauss mapping approximately planar, thereby removing the need for more complex spherical trigonometry. The signed enclosed areas are found straightforwardly 
to be

$$
\begin{aligned}
S_{1}= & S_{3}=-\frac{\beta^{2}}{4} \tan 36^{\circ} \\
S_{2}= & \frac{\beta^{2}}{2} \tan 36^{\circ}-2 \theta_{2} \sin 72^{\circ}\left(\beta-2 \theta_{1} \cos 36^{\circ}\right) \\
& \quad+2 \theta_{1} \sin 36^{\circ}\left(\theta_{1} \cos 36^{\circ}-\beta\right)+\theta_{2}^{2} \sin 144^{\circ}
\end{aligned}
$$

From $S_{1}+S_{2}+S_{3}=0$, we can solve for $\theta_{1}$ in terms of $\theta_{2}$ and $\beta$ as

$$
\theta_{1}=\frac{1}{2 \cos 36^{\circ}}\left(\sqrt{4 \theta_{2}^{2} \cos 72^{\circ}+\beta^{2}+4 \theta_{2}^{2}}-4 \theta_{2} \cos ^{2} 36^{\circ}+\beta\right)
$$

Since there are two independent variables, there is one minimised energy expression from applying Eqn 8, noting from Eqn 11:

$$
\frac{\mathrm{d} \theta_{1}}{\mathrm{~d} \theta_{2}}=2 \cos 36^{\circ}\left(\frac{2 \theta_{2}}{\sqrt{4 \theta_{2}^{2} \cos 72^{\circ}+\beta^{2}+4 \theta_{2}^{2}}}-1\right)
$$

After substituting into Eqn 8, we obtain

$$
2 \theta_{1} \cos 36^{\circ}\left(\frac{2 \theta_{2}}{\sqrt{4 \theta_{2}^{2} \cos 72^{\circ}+\beta^{2}+4 \theta_{2}^{2}}}-1\right)+\theta_{2}=0
$$

Equations 11 and 13 are now a pair of coupled, non-linear algebraic equations in $\theta_{1}$ and $\theta_{2}$ with the unique solution:

$$
\theta_{2}=0.458635 \beta \quad \theta_{1}=0.771859 \beta
$$

From the Gauss mapping in Fig. 8, the end rotation is easily read as

$$
\Psi=\theta_{1} \sin 36^{\circ}+\theta_{2} \sin 76^{\circ}=0.889875 \beta
$$

Thus, we see a linear relationship between the end rotation and the crease angle.

For the general case, the solution process can be automated using, for example, the commercial software package MATLAB [11] but with increasing solution times for large $n$. The Gauss mapping approaches a smooth curve of $4 n$ separate rotations, Fig. 9, and the ratio $\Psi / \beta$ is plotted against the total number of hinge lines, $4 n$, in Fig. 10. As expected, the ratio approaches the asymptotic result of 0.4386 from Lechenault and Adda-Bedia [3] for a singly creased disk. 


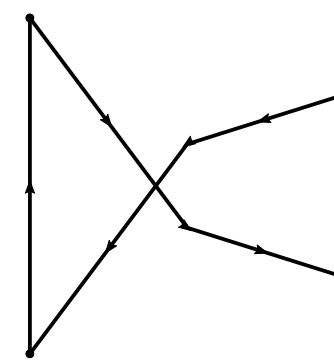

(a) $4 \times 2$ Hinges

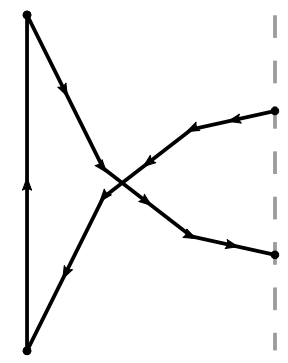

(b) $4 \times 3$ Hinges

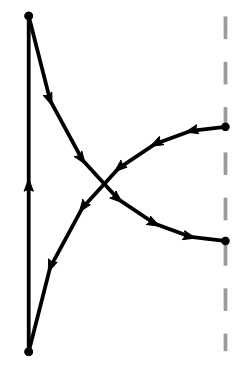

(c) $4 \times 6$ Hinges

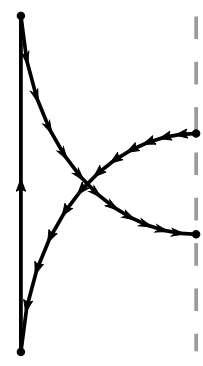

(d) $4 \times 12$ Hinges

Figure 9: Gauss mappings for 8, 12, 24, and 48 hinge line models of a creased strip. Each model is symmetric about the dashed centreline.

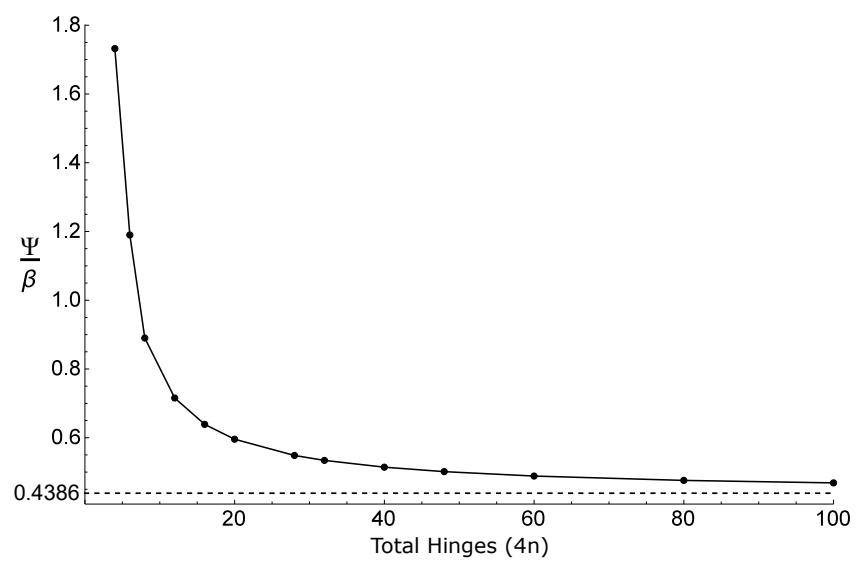

Figure 10: Ratio of end rotation to crease angle, $\Psi / \beta$, vs the number of hinge lines for a creased strip (solid) and a disk (dashed). The asymptotic limit is for a creased disk deforming continuously where $\Psi / \beta=0.4386[3]$. 
This analysis assumes that the crease sector angle, $\beta$, remains fixed during deformation. If $\beta$ were allowed to change, the Gauss mapping would have to be adjusted accordingly and the resulting end rotation would be affected. In principal this could be incorporated into the present analysis by adding an additional energy term corresponding to the change in crease sector angle. This is not straightforward since the crease sector angle will vary along the the length of the crease in practice. The length over which this transition occurs is unclear but similar behaviour has been investigated for cylindrically curved shells $[12,13]$ which may offer some insight. The fixed sector angle assumed in this study is a good approximation for the behaviour of sharply creased metallic sheets at least [14].

\section{Experimental Results}

Three sets of experiments are performed: a direct assessment of Eqn 2 by folding creases in paper card; an evaluation of the general case using folded metal strips; and an investigation of the effect of adding a hole along the crease line.

We first compare the simplest discrete model to equivalent physical specimens with $\beta=60^{\circ}$ and $\beta=20^{\circ}$ made from thick card, as shown in Fig. 11. Hinge lines are scored with a scalpel at different inclinations of $\alpha$ ranging from $20^{\circ}$ to $60^{\circ}$, and the crease angle is then set rigid by applying a thin line of resin to affirm the fixed angle assumption. The scored lines are then folded as carefully as possible without bending the flat facets but unavoidably the vertex incurs local damage. Once formed, however, all specimens are found to be bistable. The resulting end rotations are measured using a protractor, and $\Psi$ is compared to Eqn 2 in Fig. 12, where there is excellent agreement.

The end rotations for strips folded using a manual press are plotted in Fig. 13 against $\beta$ and compared to the prediction $\Psi / \beta=0.4386$. They are made either from shim steel or age-hardened copper beryllium, with thicknesses in the range $0.05 \mathrm{~mm}$ to $0.15 \mathrm{~mm}$, widths between $25 \mathrm{~mm}$ to $55 \mathrm{~mm}$, and lengths between $120 \mathrm{~mm}$ and $250 \mathrm{~mm}$. The range of materials and dimensions were chosen in 


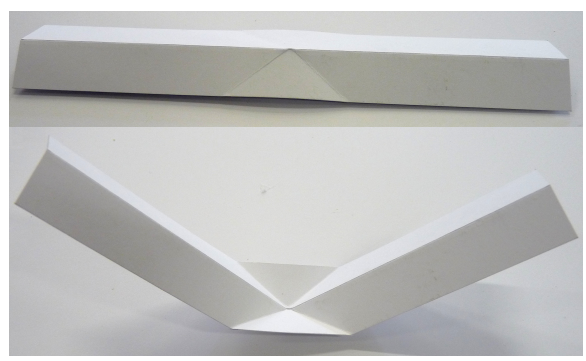

Figure 11: Four hinge line model made from paper card in its initial and inverted states.

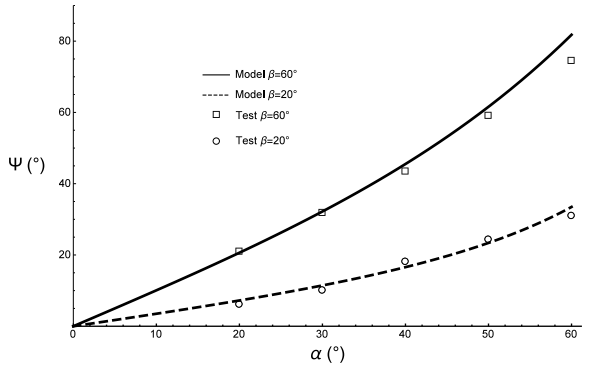

Figure 12: Comparison of Eqn 2 to experimental paper card models with hinge lines scored at various angles, $\alpha$.

order to confirm the conclusion that the deformed shape is independent of material and planform geometry. The crease sector angles of the undeformed strips were measured at each end using a protractor, and an average value taken. The strips were then carefully inverted by hand. Given the sharpness of the crease, we assumed the crease angle was unchanged, especially so for metallic strips [14]. Visually, this seemed to be reasonable but precise measurements would be difficult. Moreover, our interest is focused on the deforming material around it. The end rotation was obtained by directly measuring the angle between the two sides of the crease using a protractor and completing the triangle with two equal end rotations, as shown in Fig. 6.

For medium crease angles between $20^{\circ}$ and $40^{\circ}$ experimental results are captured rather well and no dependance on material properties or strip dimensions are apparent, as predicted. Shallower strips with $\beta$ below $20^{\circ}$ are not bistable and $\Psi$ is absent. We observe that, on pushing through, there is no softening in these cases and no clear snap. Snapping through also disappears in bistable arch beams if they are too shallow initially [9] and we surmise a similar crease angle threshold here. For $\beta$ larger than $40^{\circ}$, the experimental results begin to diverge from the prediction. This is not the result of a small-angle approximation since the results shown in Fig. 10 were obtained using a spherical Gauss mapping and are therefore valid for large angles. On closer inspection of the vertices, we do not see a well-formed vertex but a number of prominent features, including local buckling (Fig. 14b), extensive plastic deformation (Fig. 14c), and vertex 


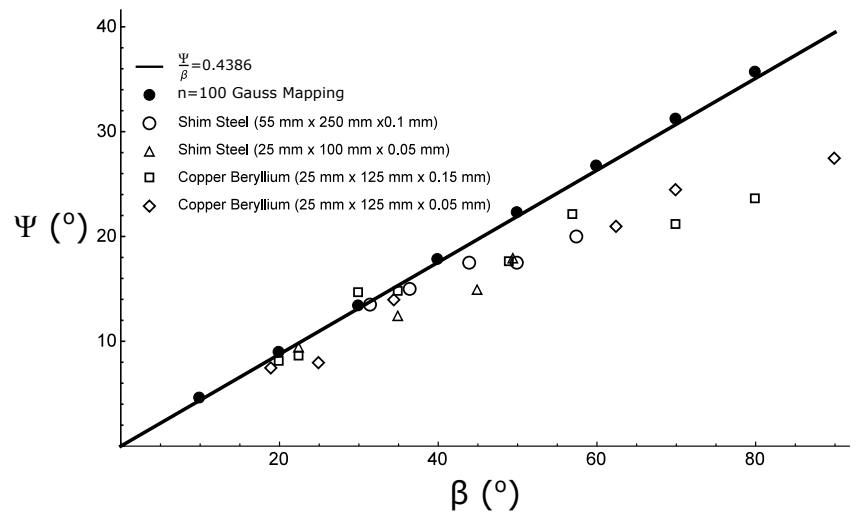

Figure 13: End rotation, $\Psi$, against crease angle, $\beta$, via experiments and theory. The solid line is the prediction from [3] of $\Psi / \beta=0.4386$. The filled circles are predictions from Sec. 2.2 using an exact Gauss Mapping calculation for $n=100$.

bifurcation (Fig. 14d), or some combination of these. The developable assumption is clearly breaking down and increasingly limiting the rotations across the vertex along the crease line for larger crease angles.

Since a precise well-formed vertex did not occur for larger $\beta$, which may be responsible for the diverging trend from the linear predictions, we added a hole to the vertex location. Strips were manufactured from $0.1 \mathrm{~mm}$ shim steel $55 \mathrm{~mm}$ wide and $250 \mathrm{~mm}$ long. Holes with diameters of $19 \mathrm{~mm}, 8 \mathrm{~mm}$ and $4 \mathrm{~mm}$ were formed in the flat strip using a standard sheet metal punch before being folded. The undeformed sector angle and end rotations were measured in the same manner as before. The deformation appears to be entirely elastic and no residual damage was observed even under repeated inversion. The end rotations for strips with an initial hole are plotted in Fig. 15. Compared to before, the results all appear to lie on the theoretical prediction of $\Psi / \beta=0.4386$ more or less without diverging at larger crease angles. There also appears to be no significant differences due to the hole size. However, when predictions of the ratio of $\Psi / \beta$ are included in Fig. 15 by using the actual hole diameters and strip dimensions in Eqn. 7 instead of using Eqn. 8, we should expect some variation-namely, higher ratios for larger holes. In practice, a hole removes the unwanted singular effects of the vertex whilst enabling the generators to 


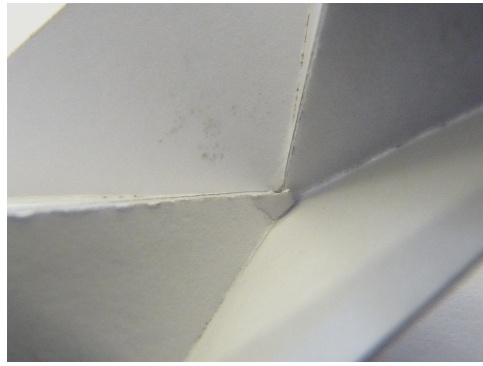

(a) Vertex damage in paper card models.

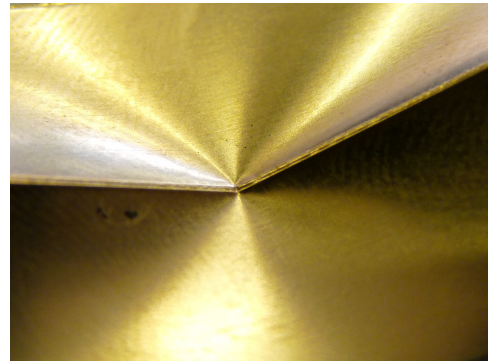

(c) Heavy plastic deformation around the vertex.

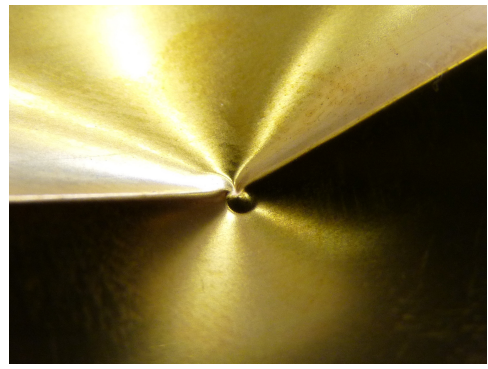

(b) Localised buckling, where a "bubble" has formed.

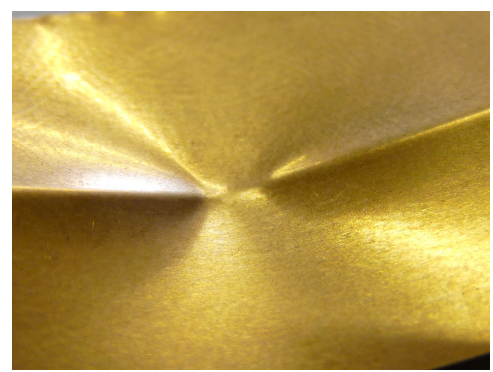

(d) Bifurcation of the vertex.

Figure 14: Non-developable deformations of the crease vertex.

be well formed beyond it; this can account for why linearity is maintained for longer with respect to $\beta$-certainly for the strips with the larger two holes of diameters $8 \mathrm{~mm}$ and $19 \mathrm{~mm}$. But a larger hole also gives greater potential variation in the local distribution of generators since they can emanate from any point within the hole, indeed, many virtual points right up to the hole boundary. This ultimately results in a configuration with lower strain energy compared to the case where they would emanate from a single central point, so we expect a marginally smaller end rotation in practice compared to theory; that this happens in our results to coincide with the prediction for no-hole is a fortuitous one given how relatively small the deviations from theory are. Also note that a slightly higher crease angle of around $30^{\circ}$ is required for strips to become bistable compared to before. Recall that bistability follows when the snapping force during push-though becomes large enough, and the presence of a hole in general reduces this force. We have been able to confirm this effect 


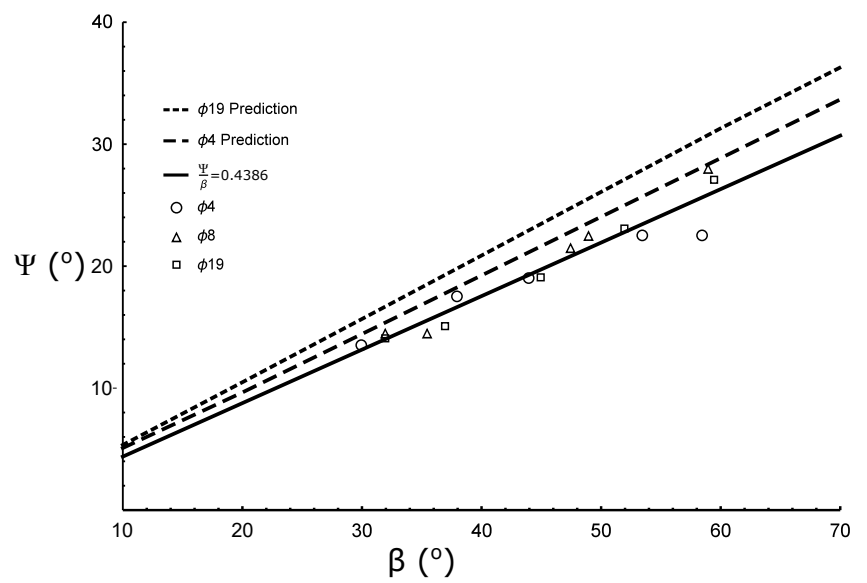

Figure 15: End rotation vs crease angle for creased strips containing a central hole on the crease line. The strips are $0.1 \mathrm{~mm}$ thick shim steel with a total length of $250 \mathrm{~mm}$ and a width of $55 \mathrm{~mm}$. Hole diameters range from $4 \mathrm{~mm}$ to $19 \mathrm{~mm}$.

using finite element analysis, which will be presented in a later study.

\section{Conclusions}

When a creased strip is bent along its axis, a snap-through change in shape occurs, often rendering a sharp bistable vertex. A rigid-facet folded approximation of this shape shows comparable gross behaviour to a creased strip. We have used a Gauss mapping technique to find the hinge rotations and the end rotation corresponding to a second geometrically compatible state, and our predictions show excellent agreement with experiments using thick card. The Gauss mapping approach was then generalised to an arbitrary number of hinge lines, in order to approach a developable continuum surface shape by minimising the total equivalent energy of bending. Experiments confirmed the predictions for medium crease angles, however, for larger crease angles the prediction and experiments diverge due to non-singular deformations at the vertex. Experiments also showed that the second stable state ceases to exist for crease angles below about $20^{\circ}$. When the crease is perforated with a hole exactly where the vertex would form, the snap-through behaviour remains without the associated damage. Formal experiments showed that the shapes of creased strips with a 


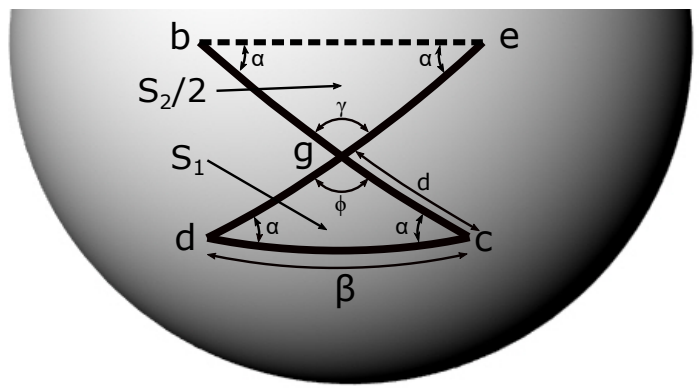

Figure A.16: Bottom half of Gauss mapping shown in Fig. 5b

hole match the predicted end rotation well for a creased strip without a hole, even for large crease angles. This suggests that the singularity at the vertex is responsible for the non-linearity seen in the experimental results for creased strips without a hole. The exact nature of the singularity and its effect on the mechanical behaviour of the strip, however, remains an open question. The approach we propose could be extended to several folded creases as per [3] and the developable deformation of other structures.

The results of this study have applications in the design of thin-walled structures. We have shown that in order for the inverted shape to form an incompatibility along the crease line must be overcome which is accomplished by localised deformation of the crease. This suggests a simple method to improve resistance to this form of damage by reinforcing the crease line. The simple bistable structure formed by a creased strip with a hole could also be used as part of a multistable structure.

\section{Appendix A. Derivation of equations 1 and 2}

In order to accommodate large rotations, the geometry of the Gauss mapping must be solved using spherical trigonometry [15]. Considering the bottom half of the Gauss mapping in Fig. 5b, shown in Fig. A.16, we solve for the areas $S_{1}$ and $S_{2}$ as well as the hinge rotations, $b c$ and $d e$, equal to $\theta$. Using the spherical law of cosines for the triangle $d c g$ :

$$
\cos \phi=\sin ^{2} \alpha \cos \beta-\cos ^{2} \alpha
$$


$d$ can be obtained using the cotangent four-part formula:

$$
\begin{aligned}
\cos \beta \cos \alpha & =\cot d \sin \beta-\cot \alpha \sin \alpha \\
\Longrightarrow \tan d & =\frac{\tan \beta / 2}{\cos \alpha}
\end{aligned}
$$

Then considering the triangle ebg and using Napier's analogy:

$$
\tan \frac{\gamma}{2}=\frac{1}{\tan \alpha \cos (\theta-d)}
$$

The areas can now be calculated:

$$
\begin{aligned}
& S_{1}=2 \alpha+\phi-\pi=2 \alpha-\pi+\arccos \left(\sin ^{2} \alpha \cos \beta-\cos ^{2} \alpha\right) \\
& S_{2}=2 \gamma+4 \alpha-2 \pi=4 \alpha-2 \pi+4 \arctan \left(\frac{1}{\tan \alpha \cos (\theta-d)}\right)
\end{aligned}
$$

Solving the condition of net zero area of the Gauss mapping given by $2 S_{1}=S_{2}$ :

$$
\tan \frac{\theta}{2}=\frac{\tan \beta / 2}{\cos \alpha}
$$

Comparing to Eqn A.2 we can immediately see that $\theta=2 d$. Using the spherical law of sines:

$$
\frac{\sin \alpha}{\sin \Psi / 2}=\frac{1}{\sin d}
$$

Therefore the end rotation $\Psi$, after substituting Eqn A.2, is:

$$
\sin \frac{\Psi}{2}=\frac{\sin \alpha \tan (\beta / 2)}{\sqrt{\cos ^{2} \alpha+\tan ^{2}(\beta / 2)}}
$$

\section{References}

[1] F. Guinot, S. Bourgeois, B. Cochelin, L. Blanchard, A planar rod model with flexible thin-walled cross-sections. Application to the folding of tape springs, International Journal of Solids and Structures 49 (1) (2012) 73-86. doi:10.1016/j.ijsolstr.2011.09.011.

[2] M. A. Dias, B. Audoly, A non-linear rod model for folded elastic strips, Journal of the Mechanics and Physics of Solids 62 (2014) 57-80. doi:10.1016/j.jmps.2013.08.012. 
[3] F. Lechenault, M. Adda-Bedia, Generic Bistability in Creased Conical Surfaces., Physical Review Letters 115 (23) (2015) 235501. doi:10.1103/PhysRevLett.115.235501.

[4] S. Farmer, C. Calladine, Geometry of developable cones, International Journal of Mechanical Sciences 47 (4-5) (2005) 509-520. doi:10.1016/j.ijmecsci.2005.02.013.

[5] K. A. Seffen, Fundamental conical defects: The d-cone, its e-cone, and its p-cone, Physical Review E - Statistical, Nonlinear, and Soft Matter Physics 94 (1) (2016) 1-8. doi:10.1103/PhysRevE.94.013002.

[6] D. Hilbert, S. Cohn-Vossen, Geometry and the Imagination, 2nd Edition, Chelsea, New York, 1990.

[7] C. Calladine, Theory of Shell Structures, 1st Edition, Cambridge University Press, Cambridge, 1983.

[8] C. Gauss, Disquisitiones generales circa superficies curvas. Gottingen; 1828. [English Translation: General Investigations of Curved Surfaces by Morehead, J.C. and Hiltebeitel, A.M.], The Princeton University Library, 1902.

[9] S. Timoshenko, D. Young, Engineering Mechanics, 4th Edition, McGrawHill, New York, 1956.

[10] V. Brunck, F. Lechenault, A. Reid, M. Adda-Bedia, Elastic theory of origami-based metamaterials, Physical Review E - Statistical, Nonlinear, and Soft Matter Physics 93 (3) (2016) 1-14. doi:10.1103/PhysRevE.93.033005.

[11] The Mathworks Inc, Matlab R2014b (2014).

[12] L. Mahadevan, A. Vaziri, M. Das, Persistence of a pinch in a pipe, Europhysics Letters (EPL) 77 (4) (2007) 40003. doi:10.1209/0295-5075/77/40003. 
[13] T. Barois, L. Tadrist, C. Quilliet, Y. Forterre, How a curved elastic strip opens, Physical Review Letters 113 (21) (2014) 1-5. doi:10.1103/PhysRevLett.113.214301.

[14] K. C. Francis, J. E. Blanch, S. P. Magleby, L. L. Howell, Origami-like creases in sheet materials for compliant mechanism design, Mechanical Sciences 4 (2) (2013) 371-380. doi:10.5194/ms-4-371-2013.

[15] G. Van Brummelen, Heavenly Mathematics: The Forgotten Art of Spherical Trigonometry, Princeton University Press, Princeton, New Jersey, 2013. 